\title{
Ecdysteroid levels in Daphnia magna during a molt cycle: Determination by radioimmunoassay (RIA) and liquid chromatography-mass spectrometry (LC-MS)
}

\author{
Dominik Martin-Creuzburg*, Stephanie A. Westerlund, Klaus H. Hoffmann \\ Department of Animal Ecology I, Universitätsstrasse 30, University of Bayreuth, 95440 Bayreuth, Germany
}

Received 12 October 2006; revised 16 November 2006; accepted 21 November 2006

Available online 12 January 2007

\begin{abstract}
Despite their abundance and their enormous significance for various ecological processes, endocrine systems of microcrustaceans have been poorly investigated. Here, we used a highly sensitive radioimmunoassay (RIA) to determine free and conjugated ecdysteroid levels in whole-body extracts of adult Daphnia magna during a complete molt cycle. Ecdysteroid levels were predominated by free ecdysteroids. Starting from basal levels in the postmolt stage the concentration of free ecdysteroids increased sharply in the early premolt stage, followed by a sharp decline back to basal levels just prior to ecdysis. Polar and apolar ecdysteroid conjugates were found in rather low amounts with little variation during the molt cycle. Only small amounts of ecdysteroids were found in newly deposited eggs of $D$. magna, which suggest a sparing investment of maternal ecdysteroids into the eggs for early embryogenesis. As in whole-body extracts, free ecdysteroids were the predominant ecdysteroids found in eggs of $D$. magna, together with small amounts of polar and intermediary amounts of apolar conjugates. Hence, the pathways leading to polar and apolar ecdysteroid conjugates appear to be of minor importance in $D$. magna. Analyses of the immunodetectable peak in free ecdysteroids by liquid chromatography-mass spectrometry (LC-MS) revealed that molting is induced most probably by an increased level of 20-hydroxyecdysone. Microcrustaceans of the genus Daphnia are key components in freshwater food webs. Examination of the functional role of ecdysteroids in controlling developmental processes might help to understand the performance of the herbivorous grazer in its environment, in particular with regard to the adverse effects of environmental xenobiotics acting as endocrine disrupters.
\end{abstract}

Keywords: Endocrinology; Microcrustaceans; Ecdysteroid conjugates; Maternal investment; Eggs; Endocrine disrupters

\section{Introduction}

Like all arthropods, crustaceans have to periodically shed their old exoskeleton in order to grow in size - a process mediated by ecdysteroids. Beside their functional role as molting hormones, ecdysteroids are involved in the control of reproduction and embryogenesis (Subramoniam, 2000). Research on ecdysteroids in crustaceans has focused almost exclusively on the Malacostraca (decapods, amphi-

\footnotetext{
* Corresponding author. Fax: +49 921552784.

E-mail address: Dominik.Martin-Creuzburg@uni-bayreuth.de Martin-Creuzburg).
}

pods, isopods); crustaceans other than malacostracans are poorly investigated.

In decapod crustaceans, the prohormone ecdysone, which is synthesized from dietary sterols, is secreted by ecdysial glands (Y-organs) and subsequently hydroxylated by target tissues to 20-hydroxyecdysone (20E; Chang and Kaufman, 2005). 20E appears to be the predominant molting hormone in crustaceans, but other ecdysteroids (together with their polar and apolar conjugates) are frequently found. Synthesis and secretion of ecdysteroids are negatively regulated by the molt-inhibiting hormone (MIH), a neuropeptide secreted by the sinus gland/X-organ complex (Chang and Kaufman, 2005). A positive control of ecdysteroid secretion is possibly mediated by the proposed 
crustacean juvenile hormone methyl farnesoate, a sesquiterpenoid related to the insect juvenile hormones, which is produced in the mandibular organ of decapod crustaceans (Subramoniam, 2000). Recent studies, however, suggest that methyl farnesoate is involved in the inhibition of ecdysteroid signaling, i.e. that it exhibits anti-ecdysteroid activity $(\mathrm{Mu}$ and LeBlanc, 2004a; Tuberty and McKenney, 2005). In insects, the ovary, the epidermis and the oenocytes are alternative sites for the synthesis of ecdysteroids (Delbecque et al., 1990). In crustaceans, the ovary accumulates significant amounts of hemolymph ecdysteroids (biosynthesis in the ovary is controversial) which are then passed into the eggs where they are presumably involved in the control of embryogenesis (Subramoniam, 2000).

Microcrustaceans of the genus Daphnia are key components in freshwater food webs; due to their abundance and their high grazing activity on the phytoplankton they provide a crucial link between primary and secondary production. Besides their ecological significance, daphnids have become important model organisms in toxicity evaluations. In particular, adverse effects of endocrine disrupting xenobiotics, such as synthetic juvenoids with anti-ecdysteroid activity (e.g. methoprene, pyriproxyfen, fenoxycarb), have been the focus of current research (e.g. $\mathrm{Mu}$ and LeBlanc, 2004a; Tuberty and McKenney, 2005). A decrease in ecdysteroid levels provoked by environmental chemicals has been found to result in developmental abnormalities in Daphnia magna embryos, which suggests that ecdysteroids are indispensable for the embryogenesis of daphnids $(\mathrm{Mu}$ and LeBlanc, 2002).

Ecdysteroids presumably involved in the shedding of embryonic membranes can either be derived from maternal sources which are passed into the eggs or, in the later embryonic stages, from endogenous synthesis when molting glands become functional ( $\mathrm{Mu}$ and LeBlanc, 2004b). Ecdysteroids that accumulate in the crustacean ovary and are then passed into the eggs are either free ecdysteroids, polar (mostly phosphate esters) or apolar (e.g. long chain fatty acid esters) ecdysteroid conjugates. These conjugates are discussed as inactive storage forms of maternally derived hormones that can be enzymatically hydrolyzed to free ecdysteroids by the developing embryo (Subramoniam, 2000).

In general, crustaceans continue to grow after sexual maturity and hence the process of molting is active throughout their lifetime. Once sexual maturity is attained, D. magna reproduces every 2-3 days, provided that growth and reproduction are not limited by unfavourable environmental conditions (e.g. low temperature, poor food conditions, crowding). At the end of each reproductive cycle, juvenile daphnids are released from the brood chamber, a process which is usually associated with the shedding of the old exoskeleton (ecdysis) and the release of new eggs from the ovaries into the brood chamber.

In the present study, we analyzed ecdysteroid levels in adult females of $D$. magna during a complete molt cycle using a high sampling frequency. Here, a molt cycle is defined as the interval between the two successive molts associated with the release of the first and second clutch neonates, respectively. Ecdysteroid levels (free, polar and apolar ecdysteroids) in whole-body extracts of adult females and in their eggs were determined by radioimmunoassay (RIA) and further characterized by liquid chromatography-mass spectrometry (LC-MS).

\section{Methods}

\subsection{Experimental set-up}

The experiments were carried out at $20^{\circ} \mathrm{C}$ and a $16 \mathrm{~h}: 8 \mathrm{~h}$ light:dark cycle with a clone of $D$. magna, which was originally isolated from Großer Binnensee, Germany (Lampert, 1991). The green alga Scenedesmus obliquus (SAG 276-3a) was used as food for the stock cultures of D. magna and for the experiments. Culture conditions and preparation of algal food suspensions are described elsewhere (Martin-Creuzburg et al., 2005). The experimental animals were pre-raised in glass beakers ( 20 animals per beaker) filled with 11 of filtered lake water $(0.2 \mu \mathrm{m}$ pore-sized membrane filter $)$ and $2 \mathrm{mg} \mathrm{Cl}^{-1}$ of $S$. obliquus until they hatched their first clutch of juveniles. Directly after the release of the juveniles, six synchronized individuals were each separated into $200 \mathrm{ml}$ of lake water containing $2 \mathrm{mg} \mathrm{Cl}^{-1}$ of $S$. obliquus. The food suspensions were renewed daily until the animals released their second clutch juveniles. Every $4 \mathrm{~h}$, five of these daphnids (three replicates) were deposited in $4 \mathrm{ml}$ of methanol, crushed with a glass bar, sonicated for $10 \mathrm{~min}$ in an ultrasonic bath, and stored at $-20^{\circ} \mathrm{C}$. The individual dry mass was estimated from the remaining animal in the experimental beakers, which was dried for $24 \mathrm{~h}$ and weighed on an electronic balance (Sartorius 4504MP8; $\pm 0.1 \mu \mathrm{g}$ ).

To analyze ecdysteroid levels in eggs of D. magna, 200-300 newly deposited eggs (harvested within $4 \mathrm{~h}$ after deposition) were dissected from the brood chambers of 20-30 females, transferred into $4 \mathrm{ml}$ of methanol, sonicated and stored at $-20^{\circ} \mathrm{C}$. For the purpose of comparison, ecdysteroid levels were also analyzed in newly laid eggs of the mediterranean field cricket Gryllus bimaculatus de Geer. Crickets were reared as described previously (Lorenz et al., 1997).

\subsection{Ecdysteroid extraction}

The ecdysteroid extraction protocol was adapted from Lafont et al. (1982) and modified according to Lorenz et al. (1997). The homogenized samples were delipidated (i.e. lipids were removed by partitioning into $3 \mathrm{ml}$ of $n$-hexane) and clarified by centrifugation $\left(6000 \mathrm{~g}, 10 \mathrm{~min}, 4^{\circ} \mathrm{C}\right)$. The methanolic supernatant was transferred into a clean test tube containing $500 \mu$ of water (HPLC-grade). The methanol was evaporated by vacuum centrifugation and the samples were redissolved in $4 \mathrm{ml}$ of water and subsequently loaded onto a $\mathrm{C}_{18}$ Sep-Pak ${ }^{\circledR}$ cartridge (Waters), which was previously rinsed with $10 \mathrm{ml}$ of methanol and equilibrated with $10 \mathrm{ml}$ of water. The ecdysteroid fractions were eluted subsequently with $4 \mathrm{ml}$ of $0 \%$, $25 \%, 60 \%$ and $100 \%$ methanol in water to separate the polar, free, and apolar conjugates. According to Thiry and Hoffmann (1992), significant amounts of apolar ecdysteroid conjugates may be present in the water washing phase $(0 \%$ methanol). Therefore, the water phase was further treated like the $100 \%$ methanol fraction eluted from the cartridge and the obtained results were combined and reported as apolar ecdysteroid conjugates. All ecdysteroid fractions were evaporated to dryness in a vacuum centrifuge. Free ecdysteroids were redissolved in $1 \mathrm{ml}$ of methanol and transferred into $2 \mathrm{ml}$ Safe-Lock ${ }^{\circledR}$ tubes (Eppendorf, Germany). Polar and apolar conjugates were redissolved in $10 \mu \mathrm{l}$ of methanol and $500 \mu \mathrm{l}$ of triethanolamine $\mathrm{HCl}-\mathrm{NaOH}$ buffer $(0.2 \mathrm{M}, \mathrm{pH} 7)$ and hydrolyzed by adding $5 \mu 1$ of a porcine liver esterase (Sigma E-2884, $250 \mathrm{U} \mathrm{mg}^{-1}$, incubation at $37^{\circ} \mathrm{C}$ for $24 \mathrm{~h}$ ). Hydrolysis was terminated with $1 \mathrm{ml}$ of methanol. The samples were clarified by centrifugation $\left(6000 \mathrm{~g}, 10 \mathrm{~min}, 4^{\circ} \mathrm{C}\right)$; the supernatant was transferred into a clean test tube, evaporated to $200 \mu \mathrm{l}$, and redissolved in $4 \mathrm{ml}$ of water. The free ecdysteroids liberated from this enzymatic 
hydrolysis step were separated by solid-phase extraction as described above (retaining only the $60 \%$ methanol fraction), evaporated to dryness and transferred into $2 \mathrm{ml}$ Eppendorf tubes with $1 \mathrm{ml}$ of methanol. Subsequently, all ecdysteroid fractions were evaporated to dryness and redissolved in $200 \mu$ RIA buffer solution $(0.1 \mathrm{M}$ boric acid, $25 \mathrm{mM}$ sodium tetraborate, $75 \mathrm{mM} \mathrm{NaCl}, \mathrm{pH} 8.4$ ).

\subsection{Radioimmunoassay}

In each sample, $100 \mu 1$ of a radiotracer solution $\left(\left[{ }^{3} \mathrm{H}\right]\right.$-ecdysone, Dupont, Bad Homburg Germany) dissolved in RIA buffer solution (180 Bq $100 \mu \mathrm{l}^{-1}$ buffer solution) was added along with $100 \mu \mathrm{l}$ of ecdysteroid antiserum (DBL-1; dilution 1:1000). The samples were vigorously vortexed and left for $2 \mathrm{~h}$ at room temperature before the reaction was stopped by adding $400 \mu \mathrm{l}$ of a saturated ammonium sulphate solution. The samples were vortexed, cooled for $20 \mathrm{~min}$ in an ice bath, and centrifugated $(15,000 \mathrm{~g}, 3 \mathrm{~min})$. The supernatant was discarded and the antibody-bound ecdysteroids were redissolved in $1.7 \mathrm{ml}$ of a $0.135: 1$ (v:v) mixture of water:scintillation fluid cocktail (Rotiszint 2211; Roth GmbH, Germany). Samples were left at room temperature overnight and then measured in a liquid scintillation analyzer (Perkin-Elmer, Tri-Carb 2800 TR). A standard curve was routinely generated with increasing amounts $(0-3 \mathrm{ng})$ of unlabelled ecdysone (Sigma) instead of sample. The overall recovery of ecdysteroids was estimated by adding $\left[{ }^{3} \mathrm{H}\right]$-ecdysone to Daphnia homogenates prior to solid-phase extraction. More than $96 \%$ of the radiolabeled hormone were recovered in the free ecdysone fraction. Since the DBL-1 antiserum appears to be specific for the steroid nucleus and crossreacts with a wide range of free ecdysteroids (see Reum and Koolman, 1989, for crossreaction profiles), the results obtained were expressed as pg ecdysteroid equivalents.

\subsection{Liquid chromatography-mass spectrometry (LC-MS)}

For LC-MS analyses, 20-30 synchronized daphnids were sampled in methanol $42-38 \mathrm{~h}$ prior to ecdysis, i.e. when immunodetectable ecdysteroid levels peaked. The samples were separated on a $150 \times 2.1 \mathrm{~mm} \mathrm{C}_{18}$ reversedphase column (ReproSil-Pur ODS-3, $5 \mu \mathrm{m}$; Dr. Maisch-GmbH, Ammerbuch, Germany) protected by a guard column (C18-cartridge, Phenomenex, Aschaffenburg, Germany) with a gradient elution of water/methanol $(\mathrm{MeOH})$ at a flow rate of $0.2 \mathrm{ml} \mathrm{min}^{-1}$ using an Eldex MicroPro HPLC coupled to an autosampler (SIL-10Advp, Shimadzu). The gradient elution involved a 30-min linear gradient $20-60 \% \mathrm{MeOH}$, an isocratic elution with $100 \% \mathrm{MeOH}$ in $5 \mathrm{~min}$, a linear gradient $60-20 \% \mathrm{MeOH}$ for $1 \mathrm{~min}$, and a 9min equilibration step at $20 \% \mathrm{MeOH}$.

MS analysis was accomplished using electrospray ionization (ESI) in the positive mode on a Shimadzu LCMS-2010A operating under the following conditions: the probe high voltage was set at $4.5 \mathrm{kV}$, nitrogen flow was $41 \mathrm{~min}^{-1}$, CDL voltage at $-20 \mathrm{~V}$ and temperature at $250^{\circ} \mathrm{C}$, the heat block at $200^{\circ} \mathrm{C}$. The samples were analyzed in the scan mode $(50-1000 \mathrm{~m} / \mathrm{z}$ at a $0.5 \mathrm{~s}$ scan interval) and by selected ion monitoring (SIM).

\section{Results}

Free ecdysteroids and their polar and apolar conjugates were determined during a complete molt cycle of $D$. magna. Ecdysteroid levels in D. magna were predominated by free ecdysteroids, which varied significantly during the molt cycle: starting from basal levels $\left(\approx 5-10 \mathrm{pgind}^{-1}\right.$ or 60 $100 \mathrm{pg} \mathrm{mg}^{-1}$ dry weight, respectively) in the postmolt stage the concentration of free ecdysteroids in D. magna culminated in the early premolt stage (up to $\approx 250 \mathrm{pg}$ ind $^{-1}$ or $2500 \mathrm{pg} \mathrm{mg}^{-1}$ dry weight, respectively; $42-38 \mathrm{~h}$ prior to ecdysis), followed by a sharp decline back to basal levels just prior to ecdysis (Fig. 1). Polar and apolar ecdysteroid
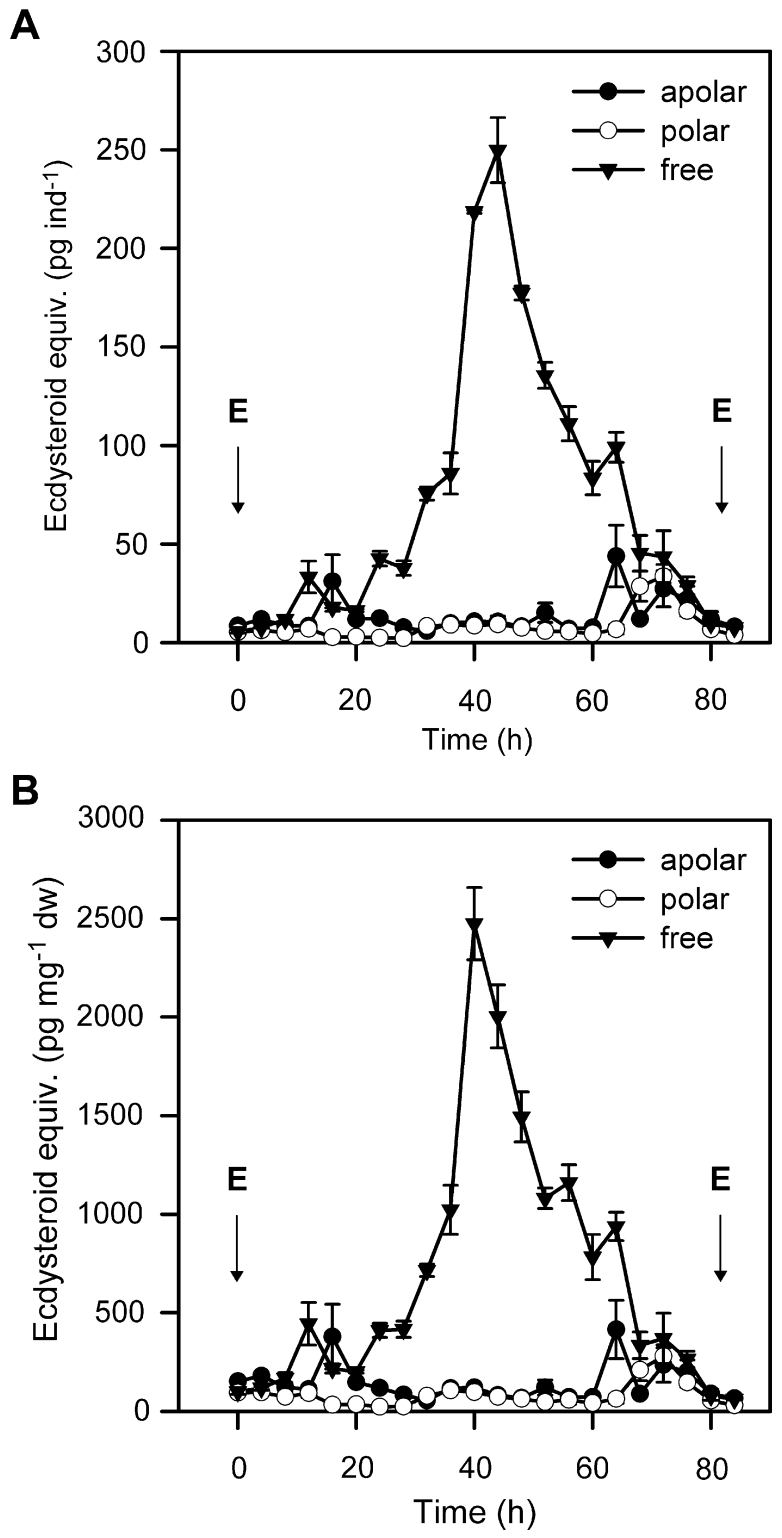

Fig. 1. Free and conjugated (polar, apolar) ecdysteroid levels in Daphnia magna collected every $4 \mathrm{~h}$ during a complete molt cycle (defined as the interval between the two successive molts associated with the release of the first and second clutch neonates, respectively). Data are means of three replicates $( \pm \mathrm{SE})$ given as pg ecdysteroid equivalents per individual (A) and as pg ecdysteroid equivalents per mg dry weight $(B)$. $(\mathrm{E}=$ ecdysis $)$.

conjugates were found in rather low amounts with little variation during the molt cycle.

Only small amounts of ecdysteroids were found in newly deposited eggs of D. magna (Fig. 2A). Free ecdysteroids were again the dominant ecdysteroids, with only small amounts of polar and intermediary amounts of apolar conjugates. The predominance of free ecdysteroids in crustacean eggs has been reported previously (e.g. Subramoniam et al., 1999); most insect species, however, accumulate polar or apolar ecdysteroids in their eggs (Espig et al., 1989 and references therein). To validate our hydrolytic procedure, newly laid eggs of the cricket $G$. bimaculatus, which are known to contain high amounts of apolar ecdysteroid con- 
A

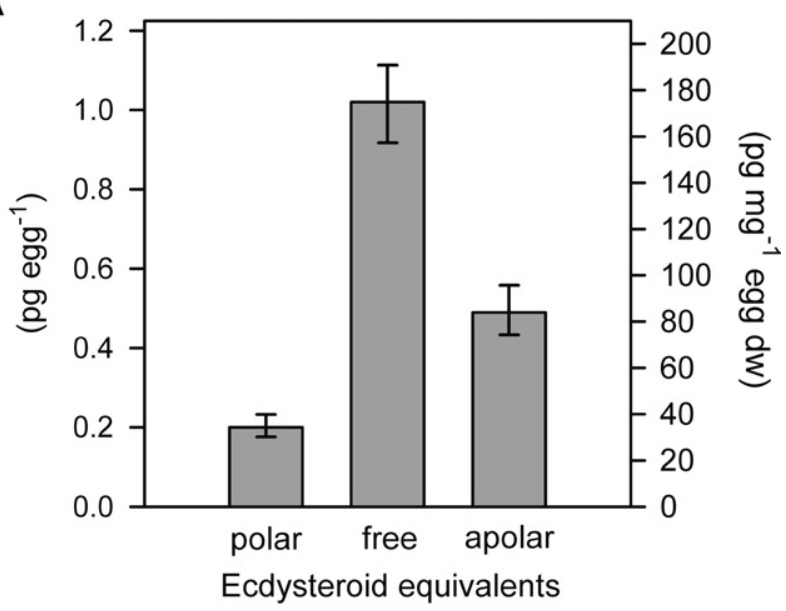

B

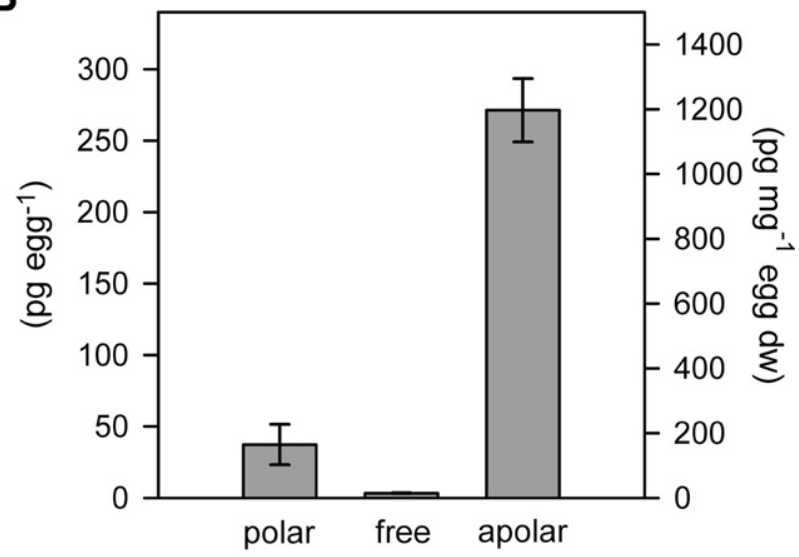

Ecdysteroid equivalents

Fig. 2. Free and conjugated (polar, apolar) ecdysteroids in newly deposited eggs of Daphnia magna (A) and Gryllus bimaculatus (B). Data are means of three replicates $( \pm \mathrm{SE})$ reported as pg ecdysteroid equivalents per egg and pg ecdysteroid equivalents per mg egg dry weight.

jugates (Espig et al., 1989), were analyzed. Thereby, 80-90\% of the detected ecdysteroids were found to be apolar conjugates, which confirmed the effectiveness of the esterase used. On a dry weight basis, eggs of $G$. bimaculatus contained 4-5 times more ecdysteroids than eggs of D. magna (Fig. 2B).

To analyze the RIA-positive ecdysteroid peak (Fig. 1), daphnids were sampled $42-38 \mathrm{~h}$ prior to ecdysis and analyzed by LC-MS. A single peak was obtained in the chromatogram when the samples were analyzed in the scan mode (Fig. 3A). Comparison of the retention time and the mass spectrum of the obtained peak (Fig. 3B) with that of authentic ecdysteroid standards suggested the presence of either 20E or its isomer inokosterone. Samples were further analyzed by SIM using masses representative for $20 \mathrm{E}$ and inokosterone $\left(\mathrm{m} / \mathrm{z}: 503.3[\mathrm{M}+\mathrm{Na}]^{+}, 519.1[\mathrm{M}+\mathrm{K}]^{+}, 535.2\right.$ $\left.\left[\mathrm{M}+3 \mathrm{H}_{2} \mathrm{O}+\mathrm{H}\right]^{+}\right)$and compared with Daphnia samples taken at the early postmolt stage (low levels of immunodetectable ecdysteroids) and spiked with standards of $20 \mathrm{E}$ and inokosterone, respectively. The relative intensity of $\mathrm{m} / \mathrm{z}$ 535.2 was found to be significantly lower $(\sim 40 \%)$ in the mass spectra of $20 \mathrm{E}$ than in mass spectra of inokosterone in
A

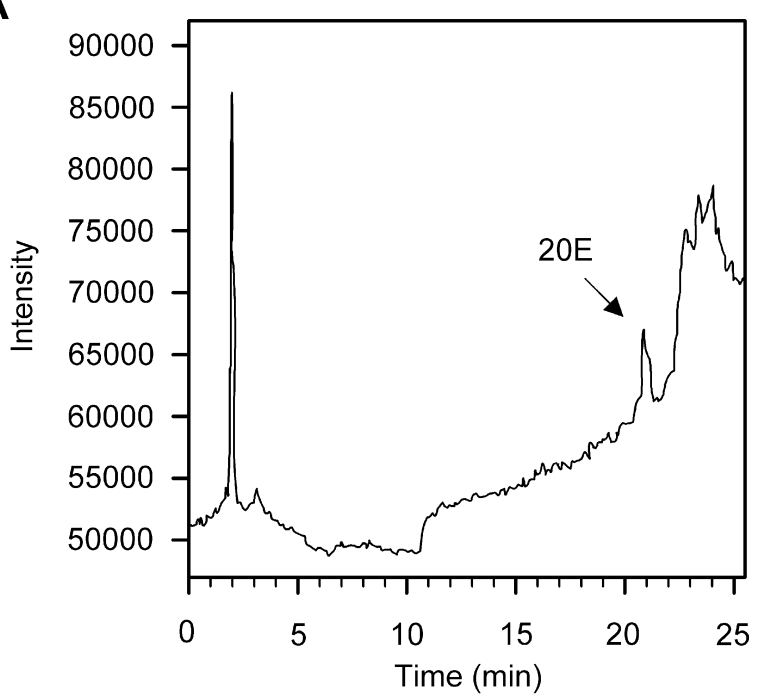

B

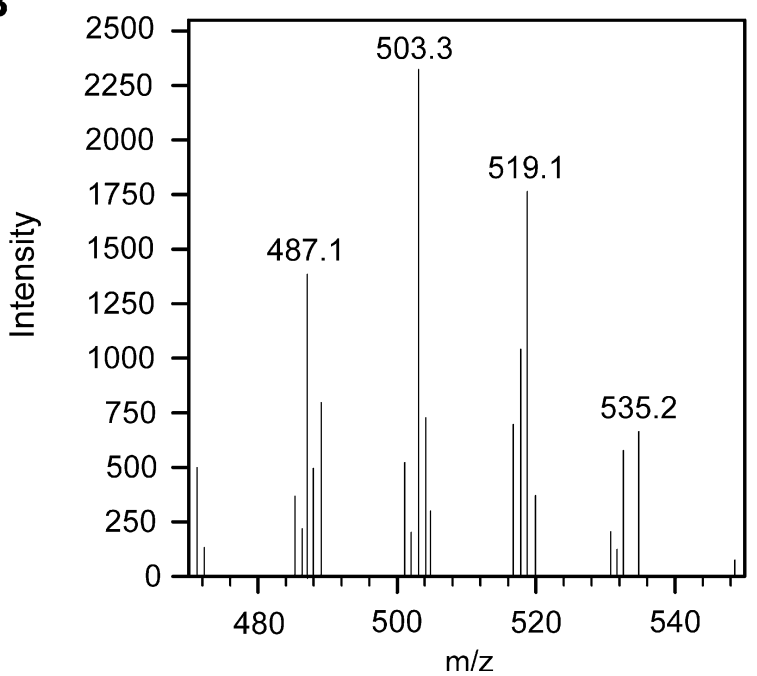

Fig. 3. Chromatogram (A) and mass spectrum (B) of 20-hydroxyecdysone (20E) in Daphnia magna sampled $40 \mathrm{~h}$ prior to ecdysis (early premolt stage). Representative masses for $20 \mathrm{E}$ adducts, $m / z: 503.3[\mathrm{M}+\mathrm{Na}]^{+}, 519.1$ $[\mathrm{M}+\mathrm{K}]^{+}, 535.2\left[\mathrm{M}+3 \mathrm{H}_{2} \mathrm{O}+\mathrm{H}\right]^{+}$.

spiked Daphnia matrix. The low relative intensity of $\mathrm{m} / \mathrm{z}$ 535.2 in the unidentified peak found in unspiked samples, together with slight differences in the retention times suggest that $20 \mathrm{E}$ is present in $D$. magna rather than inokosterone.

\section{Discussion}

Ecdysteroids are polyhydroxylated ketosteroids that serve as molting hormones in all arthropod groups. In addition, ecdysteroids are thought to be involved in the control of reproduction and embryogenesis (Subramoniam, 2000).

In Daphnia spp., endocrine systems are poorly investigated. With regard to ecdysteroids, only a few studies are available focusing mainly on exposure-effects of exogenously supplied ecdysteroids on the performance of $D$. magna. In D. magna, exposure to ecdysteroids tends to pre- 
vent successful exuviation in a dose dependent manner (Bodar et al., 1990; Baldwin et al., 2001). Bodar et al. (1990) suggested that this molting impairment is due to persistently high ecdysteroid levels in the daphnids, which may override the typical drop of ecdysteroid levels just prior to exuviation as observed in other crustaceans, including $D$. magna (Mu and LeBlanc, 2004b, and the present study). The occurrence of ecdysteroids in whole-body extracts of unexposed D. magna was first reported by Bodar et al. (1990) using an enzyme-immunoassay. Therein, ecdysteroid titres were found to fluctuate around $210 \mathrm{pg}$ ecdysteroid equivalents per $\mathrm{mg}$ dry weight $\left(\approx 80 \mathrm{pg}\right.$ ind $\left.^{-1}\right)$ in adult females. The study of Bodar et al. (1990), however, was designed to elucidate possible effect of cadmium exposure on ecdysteroid levels in D. magna, rather than to detect possible peaks of ecdysteroids which requires a much higher sampling frequency.

Ecdysteroid levels in embryos and juveniles of $D$. magna have been investigated by $\mathrm{Mu}$ and LeBlanc (2004b) using a radioimmunoassay. They reported a progressive decline in ecdysteroid levels (from $\approx 25$ to $7 \mathrm{pgind}^{-1} ; 370$ to $50 \mathrm{pg} \mathrm{mg}^{-1}$ wet tissue weight) during the first stages of embryonic development followed by a slight increase in subsequent developmental stages. This suggests that daphnid embryos rely on maternal derived ecdysteroids in their early development, and that this pool of ecdysteroids is exhausted and subsequently replenished with endogenously synthesized ecdysteroids in late embryonic development. In addition, $\mathrm{Mu}$ and LeBlanc (2004b) determined molt related ecdysteroid levels in juvenile $D$. magna and reported that ecdysteroid levels remained low ( $\approx 50 \mathrm{pg} \mathrm{mg}^{-1}$ wet tissue weight) through most of the molt cycle but increased to approximately $150 \mathrm{pg} \mathrm{mg}^{-1}$ wet tissue weigth just prior to molting.

This pattern was corroborated by the present study analyzing adult females of D. magna: starting from basal levels in the postmolt stage immunodetectable ecdysteroid levels sharply increased in the early premolt stage (42-38 h prior to ecdysis) and decreased back to basal levels just prior to ecdysis. Although absolute values of RIA-determined ecdysteroid levels are difficult to compare (Reum and Koolman, 1989), the range of concentrations of RIA-positive ecdysteroids in adult D. magna determined in the present study $\left(100-2500 \mathrm{pg} \mathrm{mg}^{-1}\right.$ dry weight) brackets the range reported by $\mathrm{Mu}$ and LeBlanc (2004b) in juvenile daphnids (500-1500 $\mathrm{pg} \mathrm{mg}^{-1}$ dry weight, calculated assuming a water content of $90 \%$ in daphnids).

Ecdysteroid levels in D. magna were predominated by free ecdysteroids, with only small amounts of polar and apolar ecdysteroid conjugates. LC-MS analyses provided evidence that an increase of $20 \mathrm{E}$ accounts for the observed RIA-peak in free ecdysteroids in the premolt stage. In decapod crustaceans, $20 \mathrm{E}$ is the predominant molting hormone. Our results strongly suggest that $20 \mathrm{E}$ is also synthesized by D. magna, and that molting in cladocerans is induced by an increased $20 \mathrm{E}$ level. The presence of small amounts of ecdysone and other free and conjugated ecdysteroids is likely but could not be confirmed in the present study.
Ecdysteroids are found in most body compartments of arthropods. The largest amounts, however, are often associated with the maturing ovary (Subramoniam, 2000). In decapods, ecdysteroids are synthesized in the Y-organ and transported through the hemolymph into the ovaries. Ecdysteroid biosynthesis in crustacean ovaries has as yet not been documented (Subramoniam, 2000); contrarily to insect ovaries which have been shown to synthesize ecdysteroids (Delbecque et al., 1990). Nevertheless, evidence has been provided that ovarian ecdysteroids are transferred into the eggs (possibly bound to the yolk protein vitellin), either as free or as conjugated (polar and/or apolar) ecdysteroids, presumably for subsequent use by the developing embryo (Subramoniam, 2000). As observed in different shrimp species (e.g. Spindler et al., 1987), total ecdysteroid levels in newly deposited eggs of D. magna were rather low (e.g. compared to total ecdysteroid levels in eggs of the cricket G. bimaculatus, Fig. 2), which suggests a sparing investment of maternal ecdysteroids into the eggs. In contrast, the comparatively high amounts of ecdysteroids found by $\mathrm{Mu}$ and LeBlanc (2004b) in early daphnid embryos $\left(\sim 25 \mathrm{pgind}^{-1}, \mathrm{Mu}\right.$ and LeBlanc; cp. to $\sim 1.7 \mathrm{pg}$ $\mathrm{egg}^{-1}$, the present study) would suggest a much higher maternal investment of ecdysteroids into the eggs, provided that no endogenous synthesis occurred in these early embryos. This discrepancy has to be further investigated to elucidate the importance of maternally derived ecdysteroids for daphnid embryogenesis.

Free ecdysteroids were the predominant ecdysteroids found in eggs of D. magna, with only small amounts of polar and intermediary amounts of apolar conjugates. The predominance of free ecdysteroids in eggs of crustaceans has been reported previously (e.g. Subramoniam et al., 1999). However, this is in contrast to many other arthropod eggs, which predominantly contain either polar (e.g. phosphate esters) or apolar (e.g. long chain fatty acid esters) conjugates rather than free ecdysteroids (cf. ecdysteroids in eggs of G. bimaculatus, Fig. 2). Ecdysteroid conjugates have been discussed as inactivation products that, if required, can be enzymatically hydrolyzed to free ecdysteroids by the developing embryo (Subramoniam, 2000). Alternatively, conjugated ecdysteroids may represent inactive end products for elimination through storage excretion; e.g. the acidic 26-oic derivatives, which often become dominant in later embryonic stages of decapod crustaceans, are possibly excreted within the egg capsule (Wilder et al., 1995). In D. magna, the pathways leading to polar or apolar ecdysteroid conjugates appear to be of minor importance, i.e. maternal ecdysteroids are transferred into the eggs predominantly in their free form. In decapod crustaceans, ecdysteroid metabolism in the eggs seems to be active throughout embryogenesis and a rapid conversion of free ecdysteroids into conjugates and vice versa has been reported (Subramoniam, 2000). Hence, the relative importance of free and conjugated ecdysteroids in eggs of $D$. magna might change with progression of embryogenesis.

In adult $D$. magna, ovarian activity is cyclic and closely synchronized with molting. Each reproductive cycle starts with the shedding of the old exoskeleton and the release of 
new eggs from the ovaries into the brood chamber. In the first hours of each reproductive cycle the ovaries are inconspicuous in size and colour. After $24 \mathrm{~h}$, the ovaries increase in size and change colours to a light green. Interestingly, these structural changes in daphnid ovaries coincide with the onset of the observed RIA-positive peak in free ecdysteroids, which may suggest a gonadotropic role of ecdysteroids in D. magna (unless the ovary itself sythesizes them). In at least some crustacean species, a close correlation between hemolymph ecdysteroid levels and ovarian maturation has been reported (Okumura et al., 1992), and it has been proposed that ecdysteroids affect vitellogenin synthesis (Chaix and De Reggi, 1982). However, a functional role of ecdysteroids in the control of vitellogenesis is controversial (cf. Subramoniam, 2000).

The significance of ecdysteroids for various developmental processes in insects and decapod crustaceans has long been recognized. However, almost nothing is known about endocrine systems in microcrustaceans; e.g. in cladocerans and copepods (see Block et al. (2003) and Johnson (2003) for ecdysteroid levels in copepods), which comprise the major zooplankton taxa. Here, for the first time, we simultaneously analyzed free and conjugated ecdysteroid levels in whole-body extracts of adult $D$. magna during a complete molt cycle. Our data clearly show that the pathways leading to conjugated ecdysteroids are of minor importance in D. magna, not only in adult animals but also in newly deposited eggs. In addition, by LC-MS analyses we provide evidence that $20 \mathrm{E}$ is the predominant molting hormone in D. magna, and that molting is induced by an increased $20 \mathrm{E}$ level.

In freshwater food webs, cladocerans of the genus Daphnia play a crucial role for the carbon transfer efficiency across the phytoplankton-zooplankton interface (Martin-Creuzburg et al., 2005, and references therein). The examination of endocrine systems in Daphnia, e.g. the functional role of ecdysteroids in controlling developmental processes, might help to understand population dynamics of the herbivorous grazer and associated effects on the carbon transfer efficiency, in particular with regard to the adverse effects of environmental xenobiotics acting as endocrine disrupters.

\section{Acknowledgments}

We are indebted to J. Koolman for providing us with ecdysteroid antiserum (DBL-1) and R. Lafont for providing us with ecdysteroid standards. We thank M.W. Lorenz and I. Dippold for RIA advice, and A. Gebhardt for experimental assistance. This work was supported by the German Research Foundation (DFG, Graduate College 678/2).

\section{References}

Baldwin, W.S., Bailey, R., Long, K.E., Klaine, S., 2001. Incomplete ecdysis is an indicator of ecdysteroid exposure in Daphnia magna. Environ. Toxicol. Chem. 20, 1564-1569.
Block, D.S., Bejarano, A.C., Chandler, G.T., 2003. Ecdysteroid concentrations through various life-stages of the meiobenthic harpacticoid copepod, Amphiascus tenuiremis and the benthic estuarine amphipod, Leptocheirus plumulosus. Gen. Comp. Endocrinol. 132, 151-160.

Bodar, C.W.M., Voogt, P.A., Zandee, D.I., 1990. Ecdysteroids in Daphnia magna: their role in moulting and reproduction and their levels upon exposure to cadmium. Aquat. Toxicol. 17, 339-350.

Chaix, J.-C., De Reggi, M., 1982. Ecdysteroid levels during ovarian development and embryogenesis in the spider crab Acanthonyx lunulatus. Gen. Comp. Endocrinol. 47, 7-14.

Chang, E.S., Kaufman, W.R., 2005. Endocrinology of Crustacea and Chelicerata. In: Gilbert, L.J. (Ed.), Comprehensive Molecular Insect Science. Elsevier, Pergamon, Amsterdam, pp. 805-842.

Delbecque, J.P., Weidner, K., Hoffmann, K.H., 1990. Alternative sites for ecdysteroid production in insects. Invertebr. Reprod. Dev. 18, 29-42.

Espig, W., Thiry, E., Hoffmann, K.H., 1989. Ecdysteroids during ovarian development and embryogenesis in the cricket, Gryllus bimaculatus de Geer. Invertebr. Reprod. Dev. 15, 143-154.

Johnson, C.L., 2003. Ecdysteroids in the oceanic copepod Calanus pacificus: variation during molt cycle and change associated with diapause. Mar. Ecol. Prog. Ser. 257, 159-165.

Lampert, W., 1991. The dynamics of Daphnia in a shallow lake. Verh. Int. Ver. Limnol. 24, 795-798.

Lafont, R., Pennetier, J.-L., Andrianjafintrimo, M., Claret, J., Modde, J.-F., Blais, C., 1982. Sample processing for high-performance liquid chromatography of ecdysteroids. J. Chromatogr. 236, 137-149.

Lorenz, J.I., Lorenz, M.W., Hoffmann, K.H., 1997. Factors regulating juvenile hormone and ecdysteroid biosynthesis in Gryllus bimaculatus (Ensifera: Gryllidae). Eur. J. Entomol. 94, 369-379.

Martin-Creuzburg, D., Wacker, A., Von Elert, E., 2005. Life history consequences of sterol availability in the aquatic keystone species Daphnia. Oecologia 144, 362-372.

Mu, X., LeBlanc, G., 2002. Environmental antiecdysteroids alter embryo development in the crustacean Daphnia magna. J. Exp. Zool. 292, 287292.

Mu, X., LeBlanc, G., 2004a. Cross communication between signaling pathways: juvenoid hormones modulate ecdysteroid activity in a crustacean. J. Exp. Zool. 301A, 793-801.

Mu, X., LeBlanc, G., 2004b. Synergistic interaction of endocrine-disrupting chemicals: model development using an ecdysone receptor antagonist and a hormone synthesis inhibitor. Environ. Toxicol. Chem. 23, 1085-1091.

Okumura, T., Han, C.H., Suzuki, Y., Aida, K., Hanyu, I., 1992. Changes in hemolymph vitellogenin and ecdysteroid levels during the reproductive and non-reproductive molt cycles in the freshwater prawn Macrobrachium nipponense. Zool. Sci. 9, 37-45.

Reum, L., Koolman, J., 1989. Radioimmunoassay of Ecdysteroids. In: Koolman, J. (Ed.), Ecdysone: From Chemistry to Mode of Action. Thieme Verlag, Stuttgart, pp. 131-143.

Spindler, K.-D., Van Wormhoudt, A., Sellos, D., Spindler-Barth, M., 1987. Ecdysteroid levels during embryogenesis in the shrimp, Palaemon serratus (Crustacea: Decapoda) - Quantitative and qualitative changes. Gen. Comp. Endocrinol. 66, 116-122.

Subramoniam, T., Tirumalai, R., Gunamalai, V., Hoffmann, K.H., 1999. Embryonic ecdysteroids in a mole crab, Emerita asiatica (MilneEdwards). J. Biosci. 24, 91-96.

Subramoniam, T., 2000. Crustacean ecdysteroids in reproduction and embryogenesis. Comp. Biochem. Physiol. C 125, 135-156.

Thiry, E., Hoffmann, K.H., 1992. Dynamics of ecdysone and 20-hydroxyecdysone metabolism after injection and ingestion in Gryllus bimaculatus (De Geer). Zool. J. Physiol. 96, 17-38.

Tuberty, S.R., McKenney Jr., C.L., 2005. Ecdysteroid responses of estuarine crustaceans exposed through complete larval development to juvenile hormone agonist insecticides. Integr. Comp. Biol. 45, 106117.

Wilder, N.M., Fusetani, N., Aida, K., 1995. The presence of 20-hydroxyecdysonic acid and ecdysonic acid in the eggs of the giant freshwater prawn Macrobrachium rosenbergii. Fish. Sci. 61, 101-106. 